\title{
Malformação de veia cava inferior e trombose venosa profunda: fator de risco de trombose venosa em jovens
}

\author{
Inferior vena cava malformation and deep venous thrombosis: \\ a risk factor of venous thrombosis in the young
}

\section{Renan Roque Onzi ${ }^{1}$, Luiz Francisco Costa ${ }^{2}$, Regis Fernando Angnes ${ }^{1}$, Luciano Amaral Domingues ${ }^{1}$, Paulo Moraes $^{3}$, Leandro Armani Scaffaro ${ }^{4}$, Carolina Mancuzo Stapenhorst ${ }^{4}$}

\section{Resumo}

A ausência da veia cava inferior, alteração no processo de formação embriológica que ocorre entre a sexta e a oitava semanas de gestação, é uma rara anomalia congênita. Porém, recentemente foi confirmada como sendo um fator de risco importante para o desenvolvimento de trombose venosa profunda, especialmente em jovens. Apresentamos um caso de trombose em veias cava inferior, ilíacas, femorais e poplíteas num jovem de 16 anos com agenesia de um segmento de veia cava infra-renal e veia renal esquerda retroaórtica.

Palavras-chave: Veia cava inferior, trombose venosa profunda.

\section{Introdução}

As malformações venosas, em especial a ausência da veia cava inferior (VCI), são achados incomuns e ocasionais durante exames complementares de abdome ou em decorrência de trombose venosa aguda da veia cava $^{1}$. Alterações no processo de formação da VCI, que ocorre embriologicamente entre a sexta e a décima semanas de gestação, podem resultar em anomalias anatômicas. Dentre as dezoito variantes já descritas, as anormalidades mais freqüentes são: duplicação da VCI, veia renal esquerda retroaórtica, transposição ou VCI esquerda e veia renal esquerda circum-aórtica ${ }^{2-4}$.

Alguns relatos ${ }^{5-7}$ têm descrito a trombose venosa profunda (TVP) em pacientes com malformações con-

\begin{abstract}
Absence of inferior vena cava, caused by aberrant development within the sixth to eighth weeks of gestation, is a rare congenital anomaly. However, it has been recently confirmed as a major risk factor for the development of deep venous thrombosis, especially in young patients. We report a case of inferior vena cava, iliac, femoral and popliteal vein thrombosis in a 16 -year-old patient with inferior vena cava agenesis and retroaortic left renal vein.
\end{abstract}

Keywords: Inferior vena cava, deep venous thrombosis.

gênitas venosas. Ruggeri et al. ${ }^{8}$ sugeriram e Chee et al. ${ }^{9}$ confirmaram que as malformações da VCI são um fator de risco importante para a TVP. A combinação de intensa atividade física e alterações anatômicas de veia cava, diminuindo a velocidade de fluxo venoso, podem aumentar a possibilidade de TVP, especialmente em jovens e atletas ${ }^{10}$.

Apresentamos um caso de trombose em veias cava inferior, ilíacas, femorais e poplíteas num jovem de 16 anos com agenesia de um segmento de veia cava infra-renal e veia renal esquerda retroaórtica.

\section{Relato do caso}

Paciente de 16 anos, masculino, negro, admitido na emergência com queixas de dificuldade de movimenta-

1. Cirurgião vascular, Instituto de Medicina Vascular, Hospital Mãe de Deus, Porto Alegre, RS.

2. Professor adjunto de Cirurgia Vascular, Universidade Federal do Rio Grande do Sul (UFRGS), Porto Alegre, RS. Cirurgião vascular, Instituto de Medicina Vascular, Hospital Mãe de Deus, Porto Alegre, RS.

3. Ecografista vascular, Laboratório de Medicina Vascular, Hospital Mãe de Deus, Porto Alegre, RS.

4. Serviço de Angiorradiologia, Hospital Mãe de Deus, Porto Alegre, RS.

Artigo submetido em 19.01.07, aceito em 25.04.07. 
ção dos membros inferiores há 24 horas. Informava emagrecimento de $14 \mathrm{~kg}$ em 4 meses, atribuído à reeducação alimentar e exercícios físicos diários. Negava outros antecedentes. Ao exame físico, apresentava aumento de volume de ambos os membros inferiores com discreto empastamento muscular de panturrilhas e coxas. Foi realizada a avaliação neurológica, inclusive com punção liquórica lombar, que foi normal. Foi solicitado eco-Doppler colorido venoso de membros inferiores que evidenciou TVP bilateral em veias poplíteas, femorais, ilíacas e VCI. A pesquisa de trombofilia (proteína S, proteína C, fator V Leiden, homocisteína, anticoagulante lúpico, anticorpos antifosfolipídeos) foi negativa.

O paciente foi heparinizado, por via endovenosa, e preparado para trombólise. Realizou-se a punção guiada por ecografia de ambas as veias poplíteas com cateteres multiperfurados $5 \mathrm{~F}$. Ambos os cateteres progrediram com facilidade até a VCI. Porém cerca de 5 centímetros acima da confluência das veias ilíacas, havia parada de progressão dos cateteres em um ponto lateral à parede da VCI (Figura 1). Realizamos flebografia, que confirmava a volumosa trombose com aumento das ilíacas e extensa circulação colateral acima destas veias, sem contrastar a VCI. Devido à hipótese de compressão extrínseca da VCI, a trombólise não foi realizada.

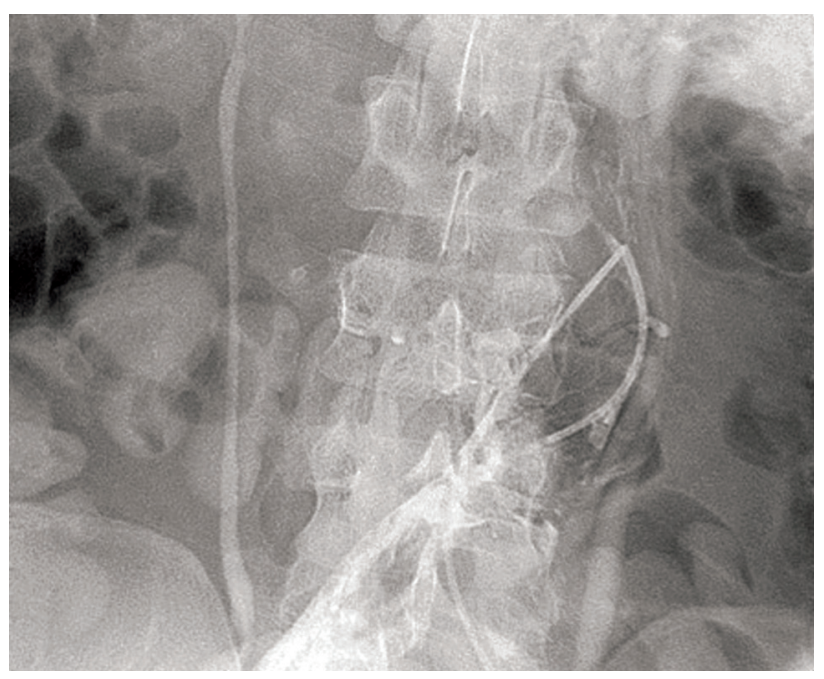

Figura 1 - Flebografia demonstrando ausência de progressão de cateteres após confluência de veias ilíacas
Solicitamos tomografia abdominal, que constatou a ausência de um segmento de veia cava inferiormente à veia renal direita, ponto onde os cateteres não progrediam durante a flebografia (Figura 2). O exame foi complementado com angiotomografia multislice (Figura 3) que evidenciou as veias ilíacas comuns formando a VCI à direita, ambas trombosadas. Porém, a VCI se continuava com a veia ázigos. Este segmento mais caudal da VCI drenava para veia renal polar esquerda com trajeto retroaórtico, formando um arco na aorta com a veia renal direita, com trajeto préaórtico. Neste segmento a veia cava era normal inclusive na VCI intra-hepática que não apresentava trombos. Não havia comunicação direta do segmento trombosado da VCI com o intra-hepático.

Diante do diagnóstico de malformação da VCI e com a melhora clínica do paciente, mesmo sem a trombólise, decidimos manter o paciente anticoagulado com warfarina e demais medidas clínicas habituais para o tratamento da TVP.

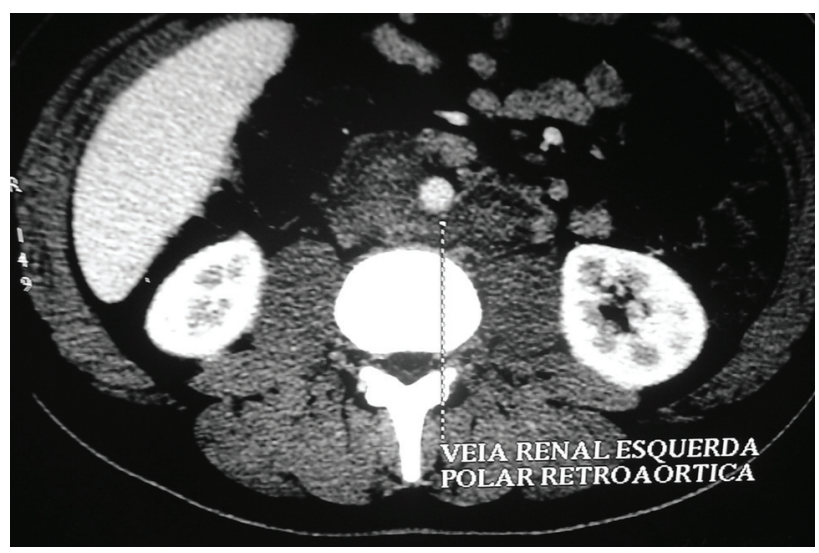

Figura 2 - Angiotomografia multislice evidenciando veia renal polar esquerda retroaórtica

\section{Discussão}

As anormalidades anatômicas da VCI são uma entidade rara causada por distúrbios do desenvolvimento ocorridos entre a sexta e décima semanas gestacionais. O desenvolvimento da VCI infra-hepática é um processo complexo que envolve três pares de veias embriogênicas: a cardinal posterior (que forma as veias ilíacas e confluentes), a subcardinal (que forma o 


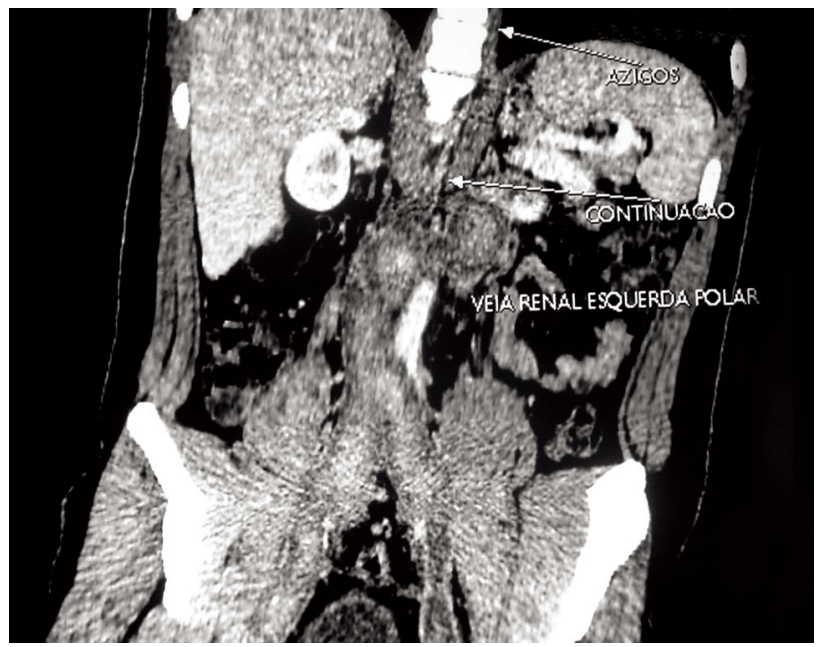

Figura 3 - Angiotomografia multislice evidenciando segmento mais caudal da VCI drenando para Veia Renal Polar Esquerda com trajeto retroaórtico, que formava um arco na aorta com a Veia Renal Direita, com trajeto pré-aórtico, que se continuava com a VCI em segmento intra-hepático

segmento renal e hepático) e a supracardinal (que forma o segmento pré-renal da VCI). As anomalias do desenvolvimento da $\mathrm{VCI}$ e veias renais foram demonstradas por muitos autores ${ }^{2-4,11,12}$.

A prevalência de malformações da VCI tem sido estimada em $0,5 \%$. Entretanto, este dado pode ser subestimado, já que tais anomalias são, em geral, assintomáticas e descobertas fortuitamente durante exames de imagem ou cirurgia do abdome $e^{9,13}$. A ausência do segmento infra-renal da VCI é uma anormalidade extremamente rara. Estudos anteriores relatam que somente $6 \%$ das anomalias da VCI ocorrem nos segmentos renal ou infra-renal ${ }^{14}$. Conforme pesquisa de Yun et al. ${ }^{15}$, até 2004 somente 21 casos comprovados por exame de imagem foram relatados na literatura de língua inglesa.

Hoje, é possível identificar os fatores de risco em cerca de $80 \%$ das TVP. Porém, nos últimos anos, alguns relatos isolados têm chamado a atenção para mais um fator de risco: as anomalias anatômicas ${ }^{9}$. Os casos relatados na literatura demonstram uma incidência entre 5 e $7 \%$ de anomalias de VCI em pacientes com TVP e uma incidência aumentada nos jovens e nas TVP bilaterais $^{8-10}$.
É notório que anormalidades da VCI podem não ser detectadas por flebografia ou eco-Doppler colorido, exames de escolha durante um episódio de $\mathrm{TVP}^{16,17}$. Esta pode ser uma das razões para que essas malformações não sejam diagnosticadas em pacientes com TVP. O screening apropriado, nos casos de TVP em jovens que atingem a região ilíaca ou femoral, deve ser conduzido com tomografia computadorizada ou ressonância magnética ${ }^{18}$.

Não há dados sobre a melhor maneira de conduzir um caso de TVP associado a anomalias de VCI quando não há outro fator de risco associado. O uso prolongado de anticoagulantes, nestes indivíduos, não está vinculado a nenhuma avaliação clínica de evidência.

O relato deste caso, associado aos demais casos publicados nos últimos anos, confirma que as alterações anatômicas de VCI são um importante fator de risco para o desenvolvimento de TVP, devendo ser sempre pesquisada em jovens.

Finalizando, reiteramos que o cirurgião vascular e o radiologista devem estar atentos a estas anormalidades vasculares. O radiologista deve distinguir entre as anomalias da VCI e outros processos patológicos do retroperitônio. $\mathrm{O}$ cirurgião vascular necessita reconhecer estas anomalias para realização de cirurgias tanto de retroperitônio quanto transperitoniais, como nas cirurgias da aorta abdominal. Da mesma forma, para a introdução dos filtros de veia cava, podemos nos deparar com a VCI duplicada e o procedimento ser inútil caso se desconheça tal anomalia.

\section{Referências}

1. Schneider JG, Eynatten MV, Dugi KA, Duex M, Nawroth PP. Recurrent deep venous thrombosis caused by congenital interruption of the inferior vena cava and heterozygous factor V Leiden mutation. J Intern Med. 2002;252:276-80.

2. Moore KL, Persaud TVN. The developing human: clinically "oriented" embryology. 5th ed. Philadelphia: Saunders; 1993. p. 309-10.

3. Kellman GM, Alpern MB, Sandler MA, Craig BM. Computed tomography of vena caval anomalies with embryologic correlation. Radiographics. 1988;8:533-56.

4. Larsen WJ. Human embryology. 3rd ed. Philadelphia: Churchill Livingstone; 2001. p. 223-4. 
5. Saito H, Sano N, Kaneda I, et al. Multisegmental anomaly of the inferior vena cava with thrombosis of the left inferior vena cava. Cardiovasc Intervent Radiol. 1995;18:410-3.

6. Zamboni P, Feo C, Pisano L, Galeotti R, Buccoliero F, Liboni A. Deep venous thrombosis in the presence of congenital hypoplasia of the inferior vena cava. J Vasc Investig. 1997;3:100-2.

7. Hamoud S, Nitecky S, Engel A, Goldsher D, Hayek T. Hypoplasia of the inferior vena cava with azygous continuation presenting as recurrent leg deep vein thrombosis. Am J Med Sci. 2000;319:414-6.

8. Ruggeri M, Tosetto A, Castaman G, Rodeghiero F. Congenital absence of the inferior vena cava: a rare risk factor for idiopathic deep-vein thrombosis. Lancet. 2001;357:441.

9. Chee YL, Culligan DJ, Watson HG. Inferior vena cava malformation as a risk factor for deep venous thrombosis in the young. Br J Haematol. 2001;114:878-80.

10. Obernosterer A, Aschauer M, Schnedl W, Lipp RW. Anomalies of the inferior vena cava in patients with iliac venous thrombosis. Ann Intern Med. 2002;136:37-41.

11. Reis RH, Esenther G. Variations in the pattern of renal vessels and their relation to the type of posterior vena cava in man. Am J Anat. 1959;104:295-318.

12. Giordano JM, Trout HH 3rd. Anomalies of the inferior vena cava. J Vasc Surg. 1986;3:924-8.
13. Eifert S, Villavicencio JL, Kao TC, Taute BM, Rich NM. Prevalence of deep venous anomalies in congenital vascular malformations of venous predominance. J Vasc Surg. 2000;31:462-71.

14. Shah NL, Shanley CJ, Prince MR, Wakefield TW. Deep venous thrombosis complicating a congenital absence of the inferior vena cava. Surgery. 1996;120:891-6.

15. Yun SS, Kim JI, Kim KH, et al. Deep venous thrombosis caused by congenital absence of inferior vena cava, combined with hyperhomocysteinemia. Ann Vasc Surg. 2004;18:124-9.

16. Prandoni P, Lensing AW, Cogo A, et al. The long-term clinical course of acute deep venous thrombosis. Ann Intern Med. 1996;125:1-7.

17. Tiesenhausen K, Amann W, Thalhammer M, Aschauer M. Aplasia of the vena cava inferior as a cause for recurring thrombosis of the lower extremities and pelvic veins. Vasa. 1999;28:289-92.

18. Thornton MJ, Ryan R, Varghese JC, Farrell MA, Lucey B, Lee MJ. A three-dimensional gadolinium-enhanced MR venography technique for imaging central veins. AJR Am J Roentgenol. 1999;173:999-1003.

Correspondência:

Renan Roque Onzi

Rua Carlos de Carvalho, 72

CEP 90630-040 - Porto Alegre, RS

Tel.: (51) 3330.5280 (51) 3230.2722

E-mail: renanonzi@cpovo.net 